
\title{
28 Research Square \\ Identifying Risk Factors and Incidence Rates for Recurrent Cesarean Scar Pregnancy: A Nested Case-Control Study
}

Sili He

Xiangya Third Hospital of Central South University

Fei Zeng

Xiangya Third Hospital of Central South University

Zhiwen Fan

Xiangya Third Hospital of Central South University

Qi Tian

Xiangya Third Hospital of Central South University

Jianfa Jiang

Xiangya Third Hospital of Central South University

Qiong Pan

Xiangya Third Hospital of Central South University

Shuijing Yi ( $\triangle 986315943 @ q q . c o m)$

Xiangya Third Hospital of Central South University

\section{Research Article}

Keywords: cesarean scar pregnancy, recurrent, risk factors

Posted Date: February 15th, 2021

DOl: https://doi.org/10.21203/rs.3.rs-184582/v1

License: (c) (i) This work is licensed under a Creative Commons Attribution 4.0 International License. Read Full License 


\section{Abstract}

Background: Cesarean scar pregnancy (CSP) is a rare and dangerous ectopic pregnancy. CSP is a late severe complication of cesarean section. In recent years, with the introduction of the comprehensive second birth policy in China, the incidence of CSP and recurrent CSP has increased. However, there are no clear data available regarding the risk factors related to recurrent CSP in the literature.

To identify risk factors and incidence rates for recurrent CSP.

Methods: A total of 1000 CSP patients were followed up for $66 \pm 19.5$ months. Among them, 86 developed recurrent CSP during the follow-up period (group 1), and the remaining without recurrence were group 2. The clinical data of these cases were reviewed in this retrospective study.

Results: The incidence rate of recurrent CSP is $8.6 \%$, and the risk factors were a history of ectopic pregnancy and prior abortions. The incidence of RCSP in patients >-35-years-old was significantly lower than that in patients $<35$-years-old $(P=0.031)$.

Conclusions: Previous ectopic pregnancy is an independent risk factor for RCSP. Additionally, the higher the number of induced abortions, the higher the incidence of RCSP.

\section{Introduction}

Cesarean scar pregnancy (CSP) is one of the long-term complications of cesarean section. It is a rare and dangerous ectopic pregnancy. The incidence rate of CSP is about $0.15 \%$ in pregnant women with a history of cesarean section and $6.1 \%$ in women experiencing ectopic pregnancies with a history of cesarean section[1]. Over the past years, there has been an increase in the incidence of CSP worldwide, especially in China. The increased prevalence of CSP might reflect the increase in cesarean delivery rates and the wide adoption of transvaginal color Doppler sonography-aided diagnosis[2]. The recent introduction of comprehensive universal second child pregnancy in China has elevated the incidence of CSP[3]. Correspondingly, the incidence of recurrent CSP has also increased.

CSP is considered to be a life-threatening condition. Embryos implant into the scar of the incision, and trophoblasts invade the connective tissue and muscular layer, causing painless vaginal bleeding and/or severe bleeding. This endangers the lives of both the patients and their fetuses and necessitates the removal of the uterus to save the patient's life. After a CSP, women with preserved fertility could attempt an intrauterine pregnancy or have recurrent CSP. However, little is known about the outcomes of subsequent pregnancies [4,5].

Currently, there is no consensus on the optimal management of CSP, a recurrent CSP might occur after conservative therapy. There are no clear data available regarding the risk factors related to recurrent CSP in the literature. Qian et al. reported that the risk factors of recurrent CSP were cesarean delivery history in rural community hospitals, thinner lower uterine segment, gestational sac bulging into the ureterovesical 
fold, history of irregular vaginal bleeding or lower abdominal pain in an earlier cesarean scar pregnancy, and early termination of the first CSP [6]. However, only 63 patients were included in the study.

Thus, we aimed to elucidate the incidence of recurrent CSP and its risk factors and speculated that this study would help the doctors to counsel the patients before a recurrence and assess the possibility of a CSP diagnosis.

\section{Methods}

We performed a retrospective study of women diagnosed with CSP at the Department of Obstetrics and Gynecology in the Third Xiangya Hospital of Central South University, People's Republic of China, from March 2007 to January 2018.Participants were informed about the purpose, methods and ethical considerations.

The inclusion criteria were as follows: Women with CSP confirmed by ultrasonography (EUB-5500, Hitachi, Japan) and beta-human chorionic gonadotropin (b-hCG) blood level >10 IU/L, indicating 30-84 days of gestation, were recruited. The diagnostic ultrasonography standard for the diagnosis of CSP was the presence of the following criteria[7]: (1) an empty uterine cavity and cervical canal, (2) development of the gestational sac in the anterior portion of the lower uterine segment, and (3) an absence of healthy myometrium between the bladder and the gestational sac. In cases where the diagnosis remained unclear after transvaginal ultrasound, pulsed Doppler ultrasonography was performed.

\section{Exclusion criteria of the study were as follows:}

1. Patients lost to follow-up; 2.Patients refused to enter the study.

All cases $(n=1000)$ were followed up for 18-148 $(66 \pm 19.5)$ months, and the clinical data of these cases were reviewed in this retrospective study. Written informed consent was obtained from each patient, and the study was approved by the Institutional Review Board of the Third Xiangya Hospital, Central South University.

The clinical characteristics of first-time CSPs of 1000 women were investigated. The continuous variables included gestational age, the interval between cesarean section and CSP, preoperative and postoperative hCG levels, gestational sac diameter, the thickness of the lower uterine segment, maternal age, number of cesarean sections, gravidity history, parity history, number of abortions, ectopic pregnancies, vaginal bleeding, lower abdominal pain, CSP classification (according to the classification by Vial et al.[8] and myometrial thickness, the endogenous type can be further divided into type I (myometrial thickness $>3 \mathrm{~mm}$ ) and type II (myometrial thickness $<3 \mathrm{~mm}$ ) and the exogenous type can be labeled as type III [9]), blood flow signals of CSP lesions, ultrasonic heartbeat, pretreatment technique used for the first-time CSP, surgical method, amount of bleeding, and use of methotrexate (MTX). Data on all the outlined variables in this study were collected from the patients for their first CSP. The default 
values for counting data were filled with average values, while the default values for the measurement data were not filled in.

The pretreatment for CSP before the operation has achieved a consensus. In the current study, pretreatment includes uterine artery embolization (UAE) coupled with MTX arterial injection[10], ultrasound-guided, high-intensity focused ultrasound (HIFU) [11], and MTX cervical injection[12]. When patients have excessive vaginal bleeding, UAE is coupled with MTX arterial injection. When patients have less vaginal bleeding or no symptoms, HIFU is administered. In the past (before January 2013), when neither method was available, MTX was injected in the cervix.

Surgical methods [13-16] include suction curettage under hysteroscopic guidance, excision of pregnancy lesions under laparoscopic guidance, excision of pregnancy lesions under laparotomy, and curettage using a myosure hysteroscopic tissue removal system. In this medical center, type I and II incision pregnancies were treated with hysteroscopic surgery, type III incision pregnancy patients underwent laparoscopic pregnancy removal.

The reproductive functions of all 1000 patients had been preserved successfully, and all patients have a documented negative HCG (<10 IU/L) following treatment.

The patients were followed up by telephone once at 6 months to enquire about their pregnancy plans, whether they were pregnant again, and whether they had recurrent CSP.

\section{Statistical analysis}

This trial was performed as a case-control study. All the data analyses were conducted using the SPSS 23.0 software program (IBM Co., Armonk, NY, USA). Statistical significance was set at $\mathrm{P}<0.05$, and the $\mathrm{P}$ values from all the tests were recorded. Descriptive statistics were used to summarize the entire population and compare the two groups of patients. We used an independent-samples t-test for continuous variable analysis if the data were normally distributed and the Mann-Whitney $U$ test if the data were non-normally distributed. The bivariate associations were evaluated using chi-square tests on the associations of the categorical data. Stratified analyses were used to assess the confounding and affecting factors. A logistic regression model was constructed using the significant variables.

\section{Results}

A total of 1000 women were enrolled in this study: 86 women with a single CSP (group I) and 914 women with recurrent CSP (group II). Recurrent CSP occurred in 86/1000 (8.6\%) patients. The patient characteristics and symptoms are presented in Tables 1 and 2. The recurrence rate (6.1\%) in patients with $\leq 3$ pregnancies $(7 / 633)$ was significantly lower than $23.2 \%$ in those with $\geq 4$ pregnancies $(78 / 336)$ $(P<0.001)$. The recurrence rate $(13.5 \%)$ of CSP in patients with multiple cesarean sections $(35 / 259)$ was significantly higher than that $(6.9 \%)$ in those with only one cesarean section $(51 / 736)(P=0.001)$. The incidence of recurrent CSP increased with the number of births $(P=0.039)$ : the incidence of recurrent CSP 
was up to $14.3 \%$ in patients with $>3$ births compared to that in patients with 1 and 2 births. Moreover, the incidence of recurrent CSP increased with the number of abortions $(P=0.014)$. The incidence of recurrent CSP in patients with previous ectopic pregnancy $(56.7 \%)$ was significantly higher than in those without previous ectopic pregnancy $(3.5 \%)(P<0.001)$. The average number of amenorrhea days and average gestational sac diameter in the recurrent CSP group were significantly lower than those in the nonrecurrence group $(P=0.006$ and $P=0.004)$. The hCG in the recurrent CSP group was significantly higher than that in the nonrecurrence group $(P=0.018)$.

Additionally, no significant difference was detected between the recurrent and the non-recurrent CSP groups with respect to the interval between cesarean section and CSP, preoperative or postoperative hCG, the thickness of the lower uterine segment, lower abdominal pain, vaginal bleeding, CSP classification, ultrasonic heartbeat, blood flow signals of CSP lesions, pretreatment technique, surgical method, use of MTX, or amount of bleeding. The incidence of recurrent CSP in patients $<35$-years-old $(9.8 \%)$ was significantly higher than that in patients $>35$-years-old $(6.4 \%)(P=0.063)$.

The positive indices of univariate analysis and clinically significant indices were included in a regression analysis. The results of the multivariable logistic regression model are presented in Table 3. A previous history of ectopic pregnancy $(P<0.001$, odds ratio $(O R)=26.565)$ and abortion $(P=0.013, O R=1.480)$ were independent risk factors for recurrent CSP, while maternal age $(P=0.031,0 R=0.437)$ was identified as a protective factor for the recurrence of CSP.

\section{Discussion}

CSP is a rare ectopic pregnancy that occurs at the site of a lower uterine segment scar leftover from an earlier cesarean delivery. With the progress of diagnosis, there has been an increase in the incidence of CSP globally in recent years[17]. The worldwide incidence of recurrent CSP reported in the literature is about $14.3 \% 15.6 \%[18,19]$. The cause of recurrent CSP is likely to be multifactorial. Risk factors that have been suggested for the occurrence of CSP include cesarean delivery history, thin lower uterine segment, gestational sac bulging into the uterovesical fold, and early termination (56 days) of the first cesarean scar pregnancy [6]. However, only a few studies reported the incidence and risk factors of recurrent CSP, and most of them include only a small sample. The present study included 1000 CSP patients who were followed up with for $66 \pm 19.54$ months, and the risk factors of recurrent CSP were evaluated using multivariate statistical analysis. The incidence rate of recurrent CSP is $8.6 \%$, and the risk factors of recurrent CSP were history of ectopic pregnancy $(\mathrm{OR}=26.565)$ and prior abortions $(\mathrm{OR}=1.480)$, and the incidence of RCSP in patients $>35$-years-old was lower than that in patients $<35$-years-old $(6.4 \%$ vs. $9.8 \%$, $\mathrm{P}=0.031$ ). The current study investigated risk factors for recurrent CSP and demonstrated several risk factors.

Previous studies suggested that the incidence of recurrent CSP is not related to patient age[6,20-22]. Conversely, the current study showed that the incidence of recurrent CSP in patients $>35$-years-old was significantly lower than that in patients $<35$-years-old $(P=0.031, O R=0.437)$ at only $6.4 \%$. This finding may 
be related to the idea that the fertility ability and desire of patients $>35 y$ ears-old are declined compared to their younger counterparts.

This research showed that abortion was an independent risk factor for recurrent CSP. The incidence of recurrent CSP was positively correlated with the number of previous induced abortions $(P=0.013$, $\mathrm{OR}=1.48$ ). The majority of the studies also speculated that the incidence of CSP is related to inflammation and injury[23]. Jauniaux et al. [24] reported that CSP might be related to unsafe abortion. Prada et al.[25] suggested that the occurrence of CSP is clearly related to a history of uterine surgery and the abnormal implantation of embryos. Lumbiganon et al. [26] found that $35 \%$ of the abortion tissues contained uterine myometrium, and thus, it was hypothesized that most abortion curettage would result in the loss of endometrium. With an increasing number of abortions, the resultant larger area of endometrial and myometrial disruption or scarring could constitute a predisposing factor in abnormal pregnancy implantation. Trophoblast adherence or invasion is enhanced when scant decidualization of the lower uterine segment is impaired by a previous myometrial disruption. The underlying mechanism explicates that scar implantation is the invasion of the myometrium through a microscopic tract that develops from the trauma of early uterine surgery, such as curettage, cesarean section, and myomectomy[27]. Moreover, an unsuitable intrauterine environment for embryo implantation makes the gestational sac more likely to implant at the scar site again.

The present study showed that a woman with a previous ectopic pregnancy was likely to have recurrent CSP $(P<0.001, O R=26.565)$. Currently, none of the studies have reported the correlation between ectopic pregnancy and CSP. Qian et al. [6] reported the incidence of RCSP in patients with previous ectopic pregnancy was higher than that in patients without previous ectopic pregnancy (4.76\% vs. $2.38 \%$ ), albeit not significantly. The main risk factor for ectopic pregnancy is a pelvic infectious disease, which is primarily caused by ascending infections. We speculated that patients with a previous history of an ectopic pregnancy might often have endometritis, which could lead to an unfavorable uterine environment for the implantation of embryos.

The present study found that the incidence of RCSP in CSP patients with more than two cesarean section times was higher than that in patients with only one cesarean section history $(13.26 \%$ versus $6.92 \%$, $P=0.073)$, albeit not significantly $(P=0.073)$. Qian et al.[6] reported that a woman with a thin lower uterine segment putatively showed RCSP. The direct cause of CSP is the existence of uterine diverticulum in the cesarean section incision, which is the local defect of the uterine wall incision or poor scar healing after cesarean section. These findings support the following hypothesis: Endometrial and myometrial disruption or scarring could be predisposing factors in abnormal pregnancy implantation. Trophoblast adherence or invasion is enhanced when scant decidualization of the lower uterine segment was impaired by a previous myometrial disruption. Typically, the uterine diverticulum caused by multiple cesarean sections was large.

Because of the limited number of reports with a large number of cases, there are no guidelines for the management of CSP or RCSP. Treatments vary from expectant management, medical management, local 
treatment, and surgical approach. In any event, early treatment will provide the best results, and most are combined treatments [28]. The present study revealed that there was no statistical difference in the incidence of recurrent CSP among different surgical groups. However, Nagi et al. [27] reported that the management of CSP is correlated with the recurrence rate of CSP. Also, laparotomy and the repair of a uterine defect might be successful in preventing recurrent CSP. However, the patient experienced recurrence even after surgical reconstruction of the cesarean scar, suggesting that no treatment can guarantee $100 \%$ safety of a subsequent pregnancy[20]. Surgical treatment and uterine repair could be complicated by poor scar healing and postoperative adhesions, which might affect the patient's fertility. Maheux-Lacroix et al.[29] analyzed a large number of studies and found that scar excision and repair were not helpful in reducing recurrent CSP occurrence.

The current study did not reveal any association between recurrent CSP and the duration of amenorrhea, the interval between CS and CSP, preoperative and postoperative hCG levels, the maximum diameter of the CSP gestational sac, the thickness of the lower uterine segment revealed by ultrasonography, gravidity history, parity history, vaginal bleeding, lower abdominal pain, number of cesarean sections, CSP classification, blood flow signals of CSP lesions, ultrasonic heartbeat, treatment technique used for the first-time CSP, or amount of bleeding. Since this is a retrospective study, some factors have not been included, and the follow-up time is relatively limited. A prolonged follow-up duration would detect additional risk factors and the correlation could be clarified.

Although recurrent CSP is rare, maintaining a high index of clinical suspicion is essential. The harm of recurrence is substantial, including uncontrolled hemorrhage and uterine rupture. Recurrent CSP does not have specific symptoms and could easily be diagnosed incorrectly[28]. Appropriate diagnostic methods, optimal treatment(s), and possible risk factors of recurrent CSP are not well-known and have not been optimized. A previous history of ectopic pregnancy and abortion is suggested as independent risk factors for recurrent CSP. The current findings regarding these and the other potential risk factors explored might aid in the prevention and early diagnosis of recurrent CSP and could be used to counsel patients at risk of recurrent CSP. Future high-quality, multicenter, large sample, randomized controlled trials are required to substantiate the current findings.

\section{Declarations}

\section{Ethics approval and consent to participate}

Written informed consent was obtained from each patient, and this study was approved by The Third Xiangya Hospital of Central South University Review Board: 2019-S430 on July 9, 2019.

The study was conducted in accordance with the Declaration of Helsinki (as revised in 2013).

\section{Consent for publication}


Consent for publication is not applicable since patient records/information was anonymized and deidentified prior to analysis.

\section{Availability of data and materials}

The data set supporting the results of this article are included within the article.

The datasets used and/or analysed during the current study are available from the corresponding author on reasonable request.

\section{Competing interests}

The authors declare that they have no conflict of interest.

\section{Funding}

This study did not receive a specific grant from any funding agency in the public, commercial, or not-forprofit sectors.

\section{Authors' contributions}

S.Y. , S.H.designed the study; Z.F., F.Z., and Q.T. conducted the follow-up; J.J. conducted the dilatation therapy and prepared the data; J.J. and Q.P. analyzed the data, S.H.drafted the manuscript. All the authors critically revised the manuscript, contributed to the final draft of the manuscript, and approved the version to be published.

\section{References}

1. Litwicka K, Greco E. Caesarean scar pregnancy: a review of management options. Curr Opin Obstet Gynecol. 2013;25:456-461.

2. Wang JH, Qian ZD, Zhuang YL, Du YJ, Zhu LH, Huang LL. Risk factors for intraoperative hemorrhage at evacuation of a cesarean scar pregnancy following uterine artery embolization. Int J Gynaecol Obstet. 2013;123:240-243.

3. Zhenyu JjZWC. To analyze the trend of cesarean section indication and situation among pregnant women during universal second child pregnancy. Chin J lab Diagn. 2020;24:64-67.

4. Jurkovic D, Hillaby K, Woelfer B, Lawrence A, Salim R, Elson CJ. First-trimester diagnosis and management of pregnancies implanted into the lower uterine segment Cesarean section scar. Ultrasound Obstet Gynecol. 2003;21:220-227.

5. Seow KM, Hwang JL, Tsai YL, Huang LW, Lin YH, Hsieh BC. Subsequent pregnancy outcome after conservative treatment of a previous cesarean scar pregnancy. Acta Obstet Gynecol Scand. 2004;83:1167-1172. 
6. Qian ZD, Guo QY, Huang LL. Identifying risk factors for recurrent cesarean scar pregnancy: a casecontrol study. Fertil Steril. 2014;102:129-134.e121.

7. Godin PA, Bassil S, Donnez J. An ectopic pregnancy developing in a previous caesarian section scar. Fertil Steril. 1997;67:398-400.

8. Vial Y, Petignat P, Hohlfeld P. Pregnancy in a cesarean scar. Ultrasound Obstet Gynecol. 2000;16:592593.

9. Group of Accounting and Family Planning, Gynecology and Obstetrics, Chinese Medical Association Expert consensus on diagnosis and treatment of Cesarean scar pregnancy. China J Obstet Gynecol. 2016;51:568-572.

10. Kutuk MS, Uysal G, Dolanbay M, Ozgun MT. Successful medical treatment of cesarean scar ectopic pregnancies with systemic multidose methotrexate: single-center experience. J Obstet Gynaecol Res. 2014;40:1700-1706.

11. Zhu X, Deng X, Xiao S, Wan Y, Xue M. A comparison of high-intensity focused ultrasound and uterine artery embolisation for the management of caesarean scar pregnancy. Int $\mathrm{J}$ Hyperthermia. 2016;32:144-150.

12. Wang M, Yang Z, Li Y, Chen B, Wang J, Ma X, et al. Conservative management of cesarean scar pregnancies: a prospective randomized controlled trial at a single center. Int $\mathrm{J}$ Clin Exp Med. 2015;8:18972-18980.

13. Bayoglu Tekin Y, Mete Ural U, Balık G, Ustuner I, Kır Şahin F, Güvendağ Güven ES. Management of cesarean scar pregnancy with suction curettage: a report of four cases and review of the literature. Arch Gynecol Obstet. 2014;289:1171-1175.

14. Hudeček R, Felsingerová Z, Felsinger M, Jandakova E. Laparoscopic Treatment of Cesarean Scar Ectopic Pregnancy. J Gynecol Surg. 2014;30:309-311.

15. Huanxiao Z, Shuqin C, Hongye J, Hongzhe X, Gang N, Chengkang X, et al. Transvaginal hysterotomy for cesarean scar pregnancy in 40 consecutive cases. Gynecol Surg. 2015;12:45-51.

16. Wu X, Xue X, Wu X, Lin R, Yuan Y, Wang Q, et al. Combined laparoscopy and hysteroscopy vs. uterine curettage in the uterine artery embolization-based management of cesarean scar pregnancy: a cohort study. Int J Clin Exp Med. 2014;7:2793-2803.

17. Shufaro Y, Nadjari M. Implantation of a gestational sac in a cesarean section scar. Fertil Steril. 2001;75:1217.

18. Wei LK, Yu LM, Mu RM, Xue FX. [Reproductive outcomes following women with previous cesarean scar pregnancy]. Zhonghua Yi Xue Za Zhi. 2018;98:2194-2197.

19. Wang CB, Tseng CJ. Primary evacuation therapy for Cesarean scar pregnancy: three new cases and review. Ultrasound Obstet Gynecol. 2006;27:222-226.

20. Holland MG, Bienstock JL. Recurrent ectopic pregnancy in a cesarean scar. Obstet Gynecol. 2008;111:541-545. 
21. Uysal F, Uysal A, Adam G. Cesarean scar pregnancy: diagnosis, management, and follow-up. J Ultrasound Med. 2013;32:1295-1300.

22. Mabuchi S, Kawase $C$, Muraji M, Kanagawa T, Kimura T. A case of recurrent cesarean scar pregnancy. Acta Obstet Gynecol Scand. 2009;88:1059-1060.

23. Timor-Tritsch IE, Monteagudo A, Santos R, Tsymbal T, Pineda G, Arslan AA. The diagnosis, treatment, and follow-up of cesarean scar pregnancy. Am J Obstet Gynecol. 2012;207:44.e41-13.

24. Jauniaux E, Jurkovic D. Placenta accreta: pathogenesis of a 20th century iatrogenic uterine disease. Placenta. 2012;33:244-251.

25. Prada E, Singh S, Villarreal C. Health consequences of unsafe abortion in Colombia, 1989-2008. Int J Gynaecol Obstet. 2012;118 Suppl 2:S92-98.

26. Lumbiganon P, Laopaiboon M, Gülmezoglu AM, Souza JP, Taneepanichskul S, Ruyan P, et al. Method of delivery and pregnancy outcomes in Asia: the WHO global survey on maternal and perinatal health 2007-08. Lancet. 2010;375:490-499.

27. Ben Nagi J, Ofili-Yebovi D, Sawyer E, Aplin J, Jurkovic D. Successful treatment of a recurrent Cesarean scar ectopic pregnancy by surgical repair of the uterine defect. Ultrasound Obstet Gynecol. 2006;28:855-856.

28. Zhang Y, Gu Y, Wang JM, Li Y. Analysis of cases with cesarean scar pregnancy. J Obstet Gynaecol Res. 2013;39:195-202.

29. Maheux-Lacroix S, Li F, Bujold E, Nesbitt-Hawes E, Deans R, Abbott J. Cesarean Scar Pregnancies: A Systematic Review of Treatment Options. J Minim Invasive Gynecol. 2017;24:915-925.

\section{Tables}

Table 1. Baseline characteristics of the participants 


\begin{tabular}{|c|c|c|c|}
\hline & Recurrent CSP & CSP & $\mathrm{P}$ \\
\hline & Group I & Group II & \\
\hline & $(n=86)$ & $(n=914)$ & \\
\hline Maternal age (years) & & & 0.063 \\
\hline 35 & $64(74.4)$ & 587 (64.2) & \\
\hline$\geq 35$ & $22(25.6)$ & 327 (35.8) & \\
\hline Gravidity history & & & 0.000 \\
\hline$\leq 3$ & $7(0.1)$ & $626(68.5)$ & \\
\hline$\geq 4$ & 79 (99.9) & 288 (31.5) & \\
\hline Number of CSs & & & 0.001 \\
\hline 1 & $51(59.3)$ & 685 (74.9) & \\
\hline$\geq 2$ & $35(40.7)$ & $229(25.1)$ & \\
\hline Parity history & & & 0.039 \\
\hline 1 & $49(57.0)$ & $617(67.5)$ & \\
\hline 2 & $31(36.0)$ & $261(28.6)$ & \\
\hline$\geq 3$ & $6(7.0)$ & $36(3.9)$ & \\
\hline Number of abortions & & & 0.014 \\
\hline 0 & $6(7.0)$ & $124(13.6)$ & \\
\hline 1 & $14(16.3)$ & $213(23.3)$ & \\
\hline 2 & $19(22.1)$ & $257(28.1)$ & \\
\hline$\geq 3$ & $47(54.6)$ & $320(35.0)$ & \\
\hline Ectopic pregnancies & & & 0.000 \\
\hline No & $31(36.0)$ & $862(94.3)$ & \\
\hline Yes & $55(64.0)$ & $52(5.7)$ & \\
\hline Gestational age (days) & $49.01 \pm 8.29$ & $52.58 \pm 11.67$ & 0.006 \\
\hline Interval between CS and CSP (years) & $6.45 \pm 4.19$ & $5.87 \pm 3.87$ & 0.186 \\
\hline
\end{tabular}

Table 2. Maternal characteristics and symptoms 


\begin{tabular}{|c|c|c|c|}
\hline & $\begin{array}{l}\text { Recurrent } \\
\text { CSP } \\
\text { Group I } \\
(n=86)\end{array}$ & $\begin{array}{l}\text { CSP } \\
\text { Group II } \\
(n=914)\end{array}$ & $P$ \\
\hline Lower abdominal pain & & & 0.797 \\
\hline Yes & $21(32.3)$ & $211(23.1)$ & \\
\hline No & $65(67.7)$ & $702(76.8)$ & \\
\hline Vaginal bleeding & & & 0.244 \\
\hline Yes & $60(69.8)$ & $580(63.5)$ & \\
\hline No & $26(30.2)$ & $334(36.5)$ & \\
\hline \multicolumn{4}{|l|}{ CSP classification } \\
\hline (Only included patients after January 2016) & 43 & 521 & 0.788 \\
\hline I & $14(32.6)$ & $167(32.1)$ & \\
\hline II & $27(62.8)$ & $315(60.5)$ & \\
\hline III & $2(4.7)$ & $39(7.5)$ & \\
\hline Ultrasonic heartbeat & & & 0.999 \\
\hline Yes & $27(31.4)$ & $287(31.4)$ & \\
\hline No & $59(68.6)$ & $627(68.6)$ & \\
\hline Blood flow signals of CSP lesions & & & 0.133 \\
\hline No signals of blood flow & $39(45.3)$ & $283(31.0)$ & \\
\hline A modicum signal of blood flow & $29(33.7)$ & $398(43.5)$ & \\
\hline Less abundant signals of blood flow & $12(14.0)$ & $172(18.8)$ & \\
\hline Abundant signals of blood flow & $6(7.0)$ & $61(6.7)$ & \\
\hline Pretreatment technique & & & 0.647 \\
\hline None & $14(16.3)$ & $134(14.7)$ & \\
\hline MTX cervical injection & $1(1.2)$ & $32(3.5)$ & \\
\hline HIFU & $41(47.7)$ & $411(45.0)$ & \\
\hline UAE & $30(34.9)$ & 337 (36.9) & \\
\hline Surgical method & & & 0.510 \\
\hline Curettage under hysteroscopic guidance & $83(96.5)$ & $886(96.9)$ & \\
\hline
\end{tabular}




\begin{tabular}{|llll|}
\hline $\begin{array}{l}\text { Excision pregnancy lesions under Laparoscopic guidance } \\
\text { Curettage under hysteroscopic Combined: laparotomy }\end{array}$ & $1(1.1)$ & $2(0.0)$ & $7(0.8)$ \\
\hline Exidance & $0(0.0)$ & $1(0.1)$ \\
\hline $\begin{array}{l}\text { Curettage under myosure Hysteroscopic tissue removal } \\
\text { system }\end{array}$ & $2(2.3)$ & $15(1.6)$ & \\
\hline Using MTX & & & \\
\hline Yes & & & \\
\hline No & $23(26.7)$ & $288(31.5)$ & \\
\hline Amount of bleeding & $63(73.3)$ & $626(68.5)$ & \\
\hline 200 mL & & & 0.361 \\
\hline 200 mL & $76(88.4)$ & $820(89.7)$ & \\
\hline Gestational age (days) & $10(11.6)$ & $94(10.3)$ & \\
\hline interval between CS and CSP (years) & $49.01 \pm 8.29$ & $52.58 \pm 11.67$ & 0.006 \\
\hline Preoperative HCG (IU/L) & $6.45 \pm 4.19$ & $5.87 \pm 3.87$ & 0.186 \\
\hline Postoperative HCG (IU/L) & $41194 \pm 49875$ & $39260 \pm 46615$ & 0.715 \\
\hline Gestational sac diameter (mm) & $15136 \pm 18669$ & $11601 \pm 12538$ & 0.018 \\
\hline Thickness of the lower uterine segments (mm) & $2.64 \pm 1.43$ & $3.13 \pm 1.52$ & 0.004 \\
\hline
\end{tabular}

Table 3 Logistic regression of the significant variables

\begin{tabular}{|lllll|}
\hline & $\mathrm{B}$ & Wald & $\mathrm{P}$ & EXP (B) $(95 \% \mathrm{Cl})$ \\
\hline Maternal age & -0.802 & 4.653 & 0.031 & $0.437(0.206-0.927)$ \\
\hline Ectopic pregnancies & 3.280 & 101.112 & 0.000 & $26.565(14.018-50.341)$ \\
\hline Number of abortions & 0.392 & 6.181 & 0.013 & $1.480(1.086-2.015)$ \\
\hline Number of CSs & 0.831 & 3.218 & 0.073 & $2.29(0.926-5.682)$ \\
\hline
\end{tabular}

